\title{
Repeated Ectopic Pregnancy after Unilateral or Bilateral Salpingectomy in Assisted Reproductive Technology
}

\author{
Qian Rong Qi, Jin Luo, Ya Qin Wang, Jing Yang and Qing Zhen Xie* \\ Reproductive Medical Center, Renmin Hospital of Wuhan University, China
}

*Corresponding author: Qing Zhen Xie, Center for Reproductive Medicine, Renmin Hospital of Wuhan University, Wuhan 430060, China.

Received Date: February 07, 2020

Published Date: February 14, 2020

\begin{abstract}
Embryo implantation occurs at the fallopian tubes, namely tubal pregnancy, is the major pattern of ectopic pregnancy. Laparoscopic salpingectomy and salpingotomy are the common ways to treat tubal pregnancies based on the damage degree of the tubes. Repeated ectopic pregnancies after unilateral or bilateral salpingectomy are rare reported but not uncommon in assisted reproductive technology (ART). This case report presents three women have had unilateral/bilateral laparoscopic salpingectomy due to at least two previous tubal pregnancies, but they experience repeated tubal pregnancies during their ART treatment. We suggest that the gynecologists should take carefully consideration on patient's future fertility when performing the surgical treatment on tubal pregnancies. Salpingectomy without proximal tube ligation potentially increase the risk of residual tubal pregnancy in ART treatment. Frozen blastocyst transfer might be an optimal option for those women with the history of repeated ectopic pregnancy. Keywords: Repeated ectopic pregnancy; Tubal pregnancy; Assisted reproductive technology
\end{abstract}

\section{Introduction}

The incidence of ectopic pregnancy has increased to a level of $2 \%$ in developed countries and may seriously affect women's health and future fertility [1]. Most of ectopic pregnancies occur in the fallopian tubes, namely tubal pregnancy. Tubal pregnancy may be life-threatening if the patients have fallopian tube rupture, heavy bleeding, or hemorrhagic shock. Tubal pregnancies can be treated by either medication or surgery. Methotrexate injection combines with mifepristone is often used to treat ectopic pregnancies that diagnosed at early stage [2]. Surgery is required when patients have viable fetus located at the fallopian tube, or at risk of tube rupture and heavy bleeding. There are two ways to remove the tubal pregnancy by laparoscopy. Salpingectomy will be performed if the damage or bleeding is severe, the affected tube will be removed with the ectopic gestational sac. However, if the damage is minimal, the ectopic gestational sac can be removed from the tube by salpingotomy and leaving the tubes in situ. However, the surgical damages may potentially cause tubal factor infertility [3].
Assisted reproductive technology (ART) has been widely applied in the treatment of infertility, especially for the women with tubal factor infertility. Patients with tubal factor infertility due to previous tubal pregnancy can be treated by in vitro fertilization and embryo transfer (IVF-ET) [4]. However, on the other hand, ART has been associated with increased ectopic pregnancy rate as high as $8.6 \%$ [5]. Repeated tubal pregnancy after unilateral/ bilateral salpingectomy is rarely reported but is not uncommon in ART. The patients suffer more painfulness and cost more expenses if they have repeated tubal pregnancy during their ART treatment. Currently, the pathogenesis of ectopic pregnancy is still unknown, and there is no effective method to prevent ectopic pregnancy.

This study presents three cases of repeated tubal pregnancy during their ART treatment. The three patients all received laparoscopic unilateral/bilateral salpingectomy or medication treatment for previous tubal pregnancies. They were diagnosed 
as tubal infertility, however, they experienced repeated tubal pregnancy during their IVF-ET treatment. One of the three cases had tubal pregnancy located at the right tube with previous pregnancy treated by methotrexate injection, the other two cases had tubal pregnancy located at the residual part of the tube which was removed by salpingectomy previously.

\section{Case Reports}

\section{Case 1}

A 31-year-old female gravid 3 para 0 with previous history of two tubal pregnancies, a left-side tubal pregnancy with her previous partner was removed via laparoscopy in 2014, a rightside tubal pregnancy with her present husband was treated by methotrexate injection combined with oral mifepristone tablets in 2016. The patient received IVF treatment and transferred two embryos in 2017 and her serum beta-hCG was 125 IU/L 12 days later. The patient was referred to our clinic with vaginal bleeding for 2 days and it has been 32 days since her embryo transfer. No intrauterine gestational sac was observed by transvaginal ultrasound, but there was a mixed mass image of $3.9 * 2.7 \mathrm{~cm}$ in the area of right fallopian tube, and a dark area at depth of 2.9 $\mathrm{cm}$ in the pelvic cavity. Her serum beta-HCG was $1520 \mathrm{IU} / \mathrm{L}$ and increased to $2280 \mathrm{IU} / \mathrm{L}$ after 48 hours. Although we explained that there is no way to prevent tubal pregnancy, the patient strongly claimed for bilateral salpingectomy or ligation to avoid repeated tubal pregnancy. During the laparoscopy, we found a right-side tubal pregnancy with active bleeding, as well as approximately $400 \mathrm{ml}$ of hemoperitoneum (Figure 1). The tubal pregnancy was removed by monopolar/bipolar electrosurgical devices plus with proximal tubal ligation on right-side tube. The pathologic diagnosis confirmed tubal pregnancy (right fallopian tube) with salpingitis. Her serum beta-HCG was decreased to 14 IU/L five days later. Two months later, this patient started a new IVF treatment cycle in our center. We performed frozen-thawed blastocyst transfer for the patient. An intrauterine pregnancy was confirmed after 30 days of transfer, the patient received C-section delivery at 39 weeks of gestation without any prenatal and perinatal complications.

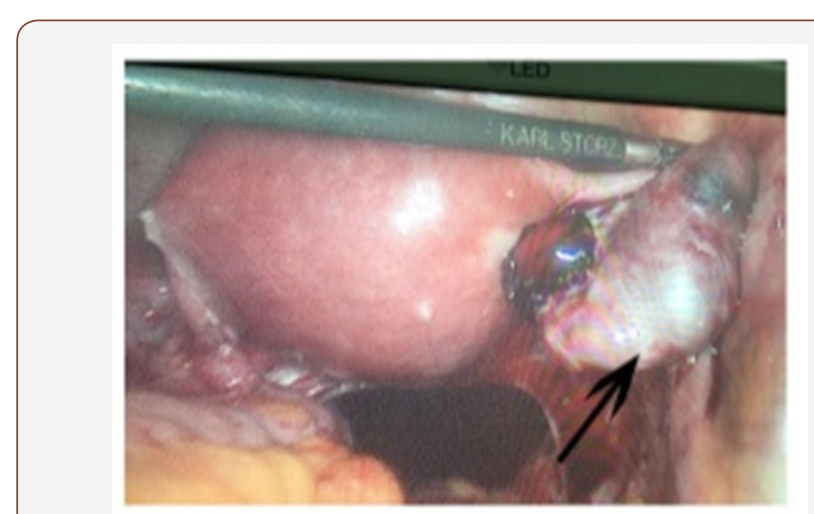

Figure 1: Tubal pregnancy in the right fallopian tube (arrow) where had a previous ectopic.

\section{Case 2}

A 36-year-old female gravid 3 para 0 with previous two tubal pregnancies, a left-side tubal pregnancy was removed by laparotomy in 2013, a right-side tubal pregnancy was removed via salpingectomy in 2015. The patient received fresh embryo transfer in 2016 and her serum beta-HCG was 197 IU/L after 12 days of embryo transfer and increased to 11,247 IU/L after 30 days of embryo transfer. No intrauterine gestational sac was observed by transvaginal ultrasound, but there was a mixed mass located at the right area of uterine adnexa. The patient didn't complain about any abdominal pain or vaginal bleeding, and she wondered why she had another ectopic pregnancy after bilateral salpingectomy. Laparoscopy revealed a protrusion located at the residual part of right tube (Figure 2). The ectopic pregnancy was removed by monopolar/bipolar electrosurgical devices. The pathologic results confirmed ectopic pregnancy with the presence of villus tissue. Her serum beta-HCG was decreased to 180 IU/L 5 days after the surgery, then decreased to $12 \mathrm{IU} / \mathrm{L}$ a week later. The patient has four frozen day-3 embryos in our center, we recommended blastocyst culture and transfer in frozen cycle. The patient received two blastocysts transfer and confirmed as single intrauterine pregnancy after 30 days of transfer. A successful full-term delivery with normal neonatal birth weight was recorded in our follow-up system.

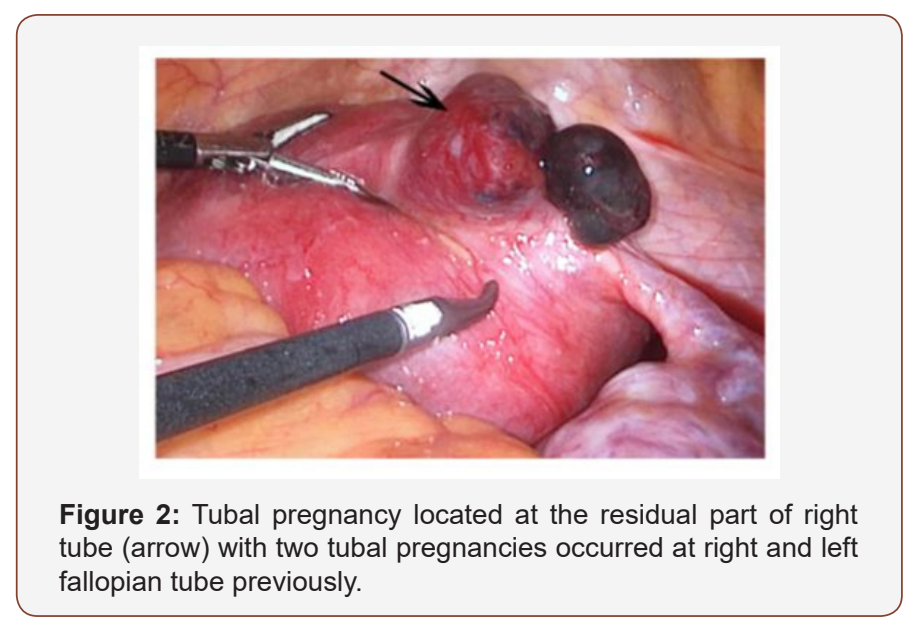

Case 3

A 35-year-old female gravid 4 para 0 with previous two tubal pregnancies and one intrauterine embryonic developmental arrest. The patient had one emergency laparoscope surgery due to left tubal pregnancy rupture and hemorrhage in 2013, followed by an intrauterine embryonic development arrest in 2014, then she was diagnosed as right-side tubal pregnancy and treated by methotrexate injection combined with oral mifepristone tablets in 2015. The patient visited our clinic and received IVF-ET treatment; her serum beta-HCG was 83 IU/L after 12 days of frozen Day 3 embryo transfer and increased to $145 \mathrm{IU} / \mathrm{L}$ after three days. No intrauterine gestational sac was found after 30 days of embryo transfer, but there was also no ultrasonic evidence for ectopic pregnancy. After thoroughly consideration and consultation, the 
patient decided to receive laparoscopic exploration and bilateral tubal proximal ligation. During the laparoscope surgery, we found that the left-side tube was missing, but the residual left tube was swelling with active bleeding (Figure 3). No abnormality was found in the right side. We removed the residual part of the left tube by monopolar/bipolar electrosurgical devices and performed bilateral proximal tube ligation. Pathologist confirmed tubal pregnancy accompanies with abortion. The patient started IVF treatment three months later, she received one cycle of frozen day- 3 embryos transfer with failed embryo implantation. We recommended blastocyst culture for the remaining three day- 3 embryos and she received one blastocyst transfer later. Currently, she received a fullterm C-section delivery without any perinatal complications.

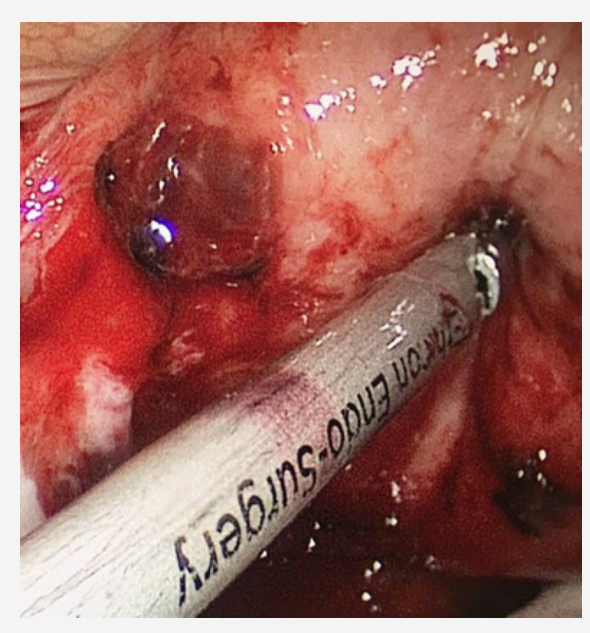

Figure 3: Tubal pregnancy located at the residual part of left tube.

\section{Discussion}

Of late, a large number of studies have been focused on the pathogenesis, risk factors, treatments, predictive biomarkers, and preventive measures for ectopic pregnancies. It has been identified that salpingitis, smoking, defective endometrial receptivity might be the risk factors for tubal pregnancy [6]. Epidemiologic investigations show that the application of ART is associated with increased rate of ectopic pregnancy, possibly because approximately $30 \%$ of infertile women are caused by tubal factor infertility, and these women share similar risk factors with ectopic pregnancies $[7,8]$. In the present study, we report that three patients with at least two previous tubal pregnancies experience repeated ectopic pregnancy during their ART treatment. The patient in Case-1 has one salpingectomy for left tubal pregnancy, followed by the medication treatment for right tubal pregnancy, she has repeated right tubal pregnancy after IVF-ET treatment. The other two cases received bilateral salpingectomy for previous tubal pregnancies, but they have repeated ectopic pregnancy located at the residual tube after embryo transfer. Currently, there is no preventive strategy for recurrent ectopic pregnancy after salpingectomy.

Ectopic pregnancy can be effectively and safely treated by either salpingectomy or salpingotomy. Randomized clinical study shows that salpingotomy experiences longer duration of the surgery and higher costs than that in salpingectomy [9]. Neither salpingectomy nor salpingotomy can prevent repeated ectopic pregnancy, although several studies report higher risk of repeated ectopic pregnancy after salpingotomy compared to salpingectomy $[3,10,11]$. The infertility rate is also increased after salpingotomy or salpingectomy due to the deficiency in tubal function. In addition, the level of Anti-Müllerian hormone, an ovarian reserve function marker is significantly lower in the salpingectomy infertility group compared with the non-salpingectomy tubal factor infertility group [12]. Therefore, there is a great chance that the patients with previous ectopic pregnancy will need to receive IVF-ET treatment. The patients suffer more stress both in physically and psychologically if they experience repeated ectopic pregnancies during their ART treatment.

In case 1 , the patient has recurrent tubal pregnancy in the right tube where the previous tubal pregnancy is treated by medication. In case 2 and case 3 , the repeated ectopic pregnancy occurs at the residual fallopian tube that left by previous salpingectomy. We notice that the residual tube is relatively long with highly risk of active bleeding. More importantly, the gynecologists didn't perform the proximal tube ligation after salpingectomy. In clinical practice, women with a history of ectopic pregnancy are concerned about how to prevent recurrent ectopic pregnancy, and some patients with two ectopic pregnancies are strongly claimed for bilateral salpingectomy or tube ligation to avoid repeated tubal pregnancy. Although bilateral salpingectomy could not eliminate the risk of interstitial or cornual pregnancy in ART treatment [13], a longer residual fallopian tube potentially increases the risk of repeated tubal pregnancy. On the other hand, previous studies suggested that excessive salpingectomy negatively affect the ovarian reserve function $[14,15]$. Therefore, we recommend that the gynecologist should inspect the tubal function thoroughly during the laparoscopy. For those women with recurrent ectopic pregnancy history, salpingectomy plus proximal tube ligation should be performed if the fallopian tubes are inelastically with inflammatory appearance.

Recently, some studies reveal that abnormal endometrial gene expression and cytokines secretion, as well as aberrant uterine receptivity are contributing to the pathogenesis of ectopic pregnancy $[16,17]$. The embryos can only implant into the endometrium where it is in the implantation window. Aberrant expression of signal networks in endometrium may lead to the shutdown of implantation window when active blastocyst entering into the uterus. On the other hand, smoking, inflammation, and infection activate the cytokines expression in fallopian tube that are essential for blastocyst adhesion and invasion, leading to the embryo implant at the fallopian tubes $[18,19]$. Several studies report that the ectopic pregnancy rate in fresh embryo transfer cycle is higher than frozen embryo transfer $[20,21]$. In addition, blastocyst transfer may be an effective method to prevent ectopic pregnancy since the endometrium is more receptive to blastocyst, 
while the embryo on day 3 may be more inclined to enter into fallopian tube [5]. In our study, three patients have failed history during their fresh cycle or day 3 embryo transfer cycle. They all achieved successful pregnancy by blastocyst transfer in frozen cycle. Therefore, frozen blastocyst transfer might be able to improve the outcomes in women with unsatisfactory pregnant history.

In conclusion, the pathogenesis of ectopic pregnancy is still unknown. The gynecologists should consider about patients' future fertility when performing laparoscopic salpingectomy in tubal pregnancy surgeries. Salpingectomy without proximal tub ligation potentially increase the risk of tubal pregnancy at the residual tubes. Frozen blastocyst transfer might be the optimal option for women with the history of repeated ectopic pregnancies.

\section{Acknowledgement}

None.

\section{Conflict of Interest}

Authors declare no conflict of interest.

\section{References}

1. Panelli DM, Phillips CH, Brady PC (2015) Incidence, diagnosis and management of tubal and nontubal ectopic pregnancies: a review. Fertil Res Pract 1: 15.

2. Marret H, Fauconnier A, Dubernard G, Misme H, Lagarce L, et al. (2016) Overview and guidelines of off-label use of methotrexate in ectopic pregnancy: report by CNGOF. Eur J Obstet Gynecol Reprod Biol 205: 105-109.

3. Chen L, Zhu D, Wu Q, Yu Y (2017) Fertility outcomes after laparoscopic salpingectomy or salpingotomy for tubal ectopic pregnancy: A retrospective cohort study of 95 patients. Int J Surg 48: 59-63.

4. Revel A, Ophir I, Koler M, Achache H, Prus D (2008) Changing etiology of tubal pregnancy following IVF. Hum Reprod 23(6): 1372-1376.

5. Santos-Ribeiro S, Tournaye H, Polyzos NP (2016) Trends in ectopic pregnancy rates following assisted reproductive technologies in the UK: a 12-year nationwide analysis including 160000 pregnancies. Hum Reprod 31(2): 393-402.

6. Farquhar CM (2005) Ectopic pregnancy. Lancet 366(9485): 583-591.

7. Li Z, Sullivan EA, Chapman M, Farquhar C, Wang YA (2015) Risk of ectopic pregnancy lowest with transfer of single frozen blastocyst. Hum Reprod 30(9): 2048-2054.
8. Perkins KM, Boulet SL, Kissin DM, Jamieson DJ (2015) Risk of ectopic pregnancy associated with assisted reproductive technology in the United States, 2001-2011. Obstet Gynecol 125(1): 70-78.

9. Mol F, van Mello NM, Strandell A, Jurkovic D, JA Ross, et al. (2015) Costeffectiveness of salpingotomy and salpingectomy in women with tubal pregnancy (a randomized controlled trial). Hum Reprod 30(9): 20382047.

10. Li J, Jiang K, Zhao F (2015) Fertility outcome analysis after surgical management of tubal ectopic pregnancy: a retrospective cohort study. BMJ Open 5: e007339.

11. Cheng X, Tian X, Yan Z, Jia M, Deng J, et al. (2016) Comparison of the Fertility Outcome of Salpingotomy and Salpingectomy in Women with Tubal Pregnancy: A Systematic Review and Meta-Analysis. PLoS One 11: e0152343.

12. Grynnerup AG, Lindhard A, Sørensen S (2013) Anti-Mullerian hormone levels in salpingectomized compared with nonsalpingectomized women with tubal factor infertility and women with unexplained infertility. Acta Obstet Gynecol Scand 92(11): 1297-1303.

13. Oki T, Douchi T, Nakamura S, Maruta K, Ijuin H, et al. (1998) A woman with three ectopic pregnancies after in-vitro fertilization and embryo transfer. Hum Reprod 13(2): 468-470.

14. Yuan Z, Cao D, Bi X, Yu M, Yang J, et al. (2019) The effects of hysterectomy with bilateral salpingectomy on ovarian reserve. Int J Gynaecol Obstet 145(2): 233-238.

15. Ye XP, Yang YZ, Sun XX (2015) A retrospective analysis of the effect of salpingectomy on serum antiMullerian hormone level and ovarian reserve. Am J Obstet Gynecol 212(1): 53.

16. Krishnan T, Winship A, Sonderegger S, Menkhorst, Horne AW, et al. (2013) The role of leukemia inhibitory factor in tubal ectopic pregnancy. Placenta 34(11): 1014-1019.

17. Li HW, Liao SB, Chiu PC, Yeung WB, Ng EH, et al. Effects of adrenomedullin on the expression of inflammatory cytokines and chemokines in oviducts from women with tubal ectopic pregnancy: an in-vitro experimental study. Reprod Biol Endocrinol 13: 120.

18. Taylor AH, Ang C, Bell SC, Konje JC (2007) The role of the endocannabinoid system in gametogenesis, implantation and early pregnancy. Hum Reprod Update 13(5): 501-513.

19. Ji YF, Chen LY, Xu KH, Yao JF, Shi YF (2009) Locally elevated leukemia inhibitory factor in the inflamed fallopian tube resembles that found in tubal pregnancy. Fertil Steril 91(6): 2308-2314.

20. Londra L, Moreau C, Strobino D, Garcia J, Zacur H, et al. (2015) Ectopic pregnancy after in vitro fertilization: differences between fresh and frozen-thawed cycles. Fertil Steril 104(1): 110-118.

21. Ozgur K, Berkkanoglu M, Bulut H, Humaidan P, Coetzee K (2015) Perinatal outcomes after fresh versus vitrified-warmed blastocyst transfer: retrospective analysis. Fertil Steril 104(4): 899-907. 